\title{
Correction to: New Therapies to Reduce Intraocular Pressure, by Konda, S.M. and Kaufman, P.L. J Ocul Pharmacol Ther 2019;35(6):322-324. DOI: 10.1089/jop.2019.0059
}

IN THE July/August 2019 issue of Journal of Ocular Pharmacology and Therapeutics (vol. 35, no. 6; 322-324) the article entitled New Therapies to Reduce Intraocular Pressure by Konda and Kaufman requires correction. The title of the article and the legends of Figures 1 and 2 have been updated.

The title of the article originally read:

New Therapies to Reduce Intraocular Pressures

This has been corrected to read:

New Therapies to Reduce Intraocular Pressure

In addition, on page 323, the figure legend for Fig. 1 was originally listed as:

FIG. 1. Schematic representation of the structural aspects of cellular contractility and relaxation (courtesy of Dr. Benjamin Geiger). When the actin-myosin system of the JCT cells and the SCIW endothelial cells contract in synchrony, the entire JCT complex is tightened and stiffened, and SC is narrowed, increasing outflow resistance and raising IOP. When the actin-myosin system relaxes, the JCT complex expands and SC dilates, reducing outflow resistance. IOP, intraocular pressure; JCT, juxtacanalicular TM; SC, Schlemm's canal; SCIW, Schlemm's canal inner wall; TM, trabecular meshwork.

This has been corrected to read:

FIG. 1. Schematic representation of the structural aspects of cellular contractility and relaxation (courtesy of Dr. Benjamin Geiger). When the actin-myosin system of the JCT cells and the SCIW endothelial cells contract in synchrony, the entire JCT complex is tightened and stiffened, and SC is narrowed, increasing outflow resistance and raising IOP. When the actinmyosin system relaxes, the JCT complex expands and SC dilates, reducing outflow resistance. Reprinted from Cell, 110 by Geiger B, Bershadsky A. Exploring the neighborhood: adhesion-coupled cell mechanosensors. Pages 139-142. Copyright 2002, with permission from Elsevier.

IOP, intraocular pressure; JCT, juxtacanalicular TM; SC, Schlemm's canal; SCIW, Schlemm's canal inner wall; TM, trabecular meshwork.

Also on page 323, the figure legend for Fig. 2 was originally listed as:

The ROCK signaling pathway and the calcium-calmodulin MLCK pathway regulate the actin-myosin contractility system. Stimulation of these pathways induces cellular contractility; inhibition of these pathways leads to cellular relaxation, disassembly of the actin microfilament system, and degradation and weakening of cell-ECM adhesions (focal contacts). Consequently, both Rho kinase/ROCK inhibitors and NO relax and expand the JCT and dilate SC, decreasing aqueous humor outflow resistance and reducing IOP. Y-27632, a paradigm ROCK inhibitor, acts "mid-way" on the system, whereas NO likely inhibits it upstream. ECM, extracellular matrix; NO, nitric oxide; MLCK, myosin light chain kinase; ROCK, Rhoassociated protein kinase. 
This has been corrected to read:

The ROCK signaling pathway and the calcium-calmodulin MLCK pathway regulate the actin-myosin contractility system. Stimulation of these pathways induces cellular contractility; inhibition of these pathways leads to cellular relaxation, disassembly of the actin microfilament system, and degradation and weakening of cell-ECM adhesions (focal contacts). Consequently, both Rho kinase/ROCK inhibitors and NO relax and expand the JCT and dilate SC, decreasing aqueous humor outflow resistance and reducing IOP. Y-27632, a paradigm ROCK inhibitor, acts "mid-way" on the system, whereas NO likely inhibits it upstream. Figure adapted/updated from Alexander Bershadsky with permission.

ECM, extracellular matrix; NO, nitric oxide; MLCK, myosin light chain kinase; ROCK, Rho-associated protein kinase.

The online version of the article has been corrected. The authors apologize for the error. 